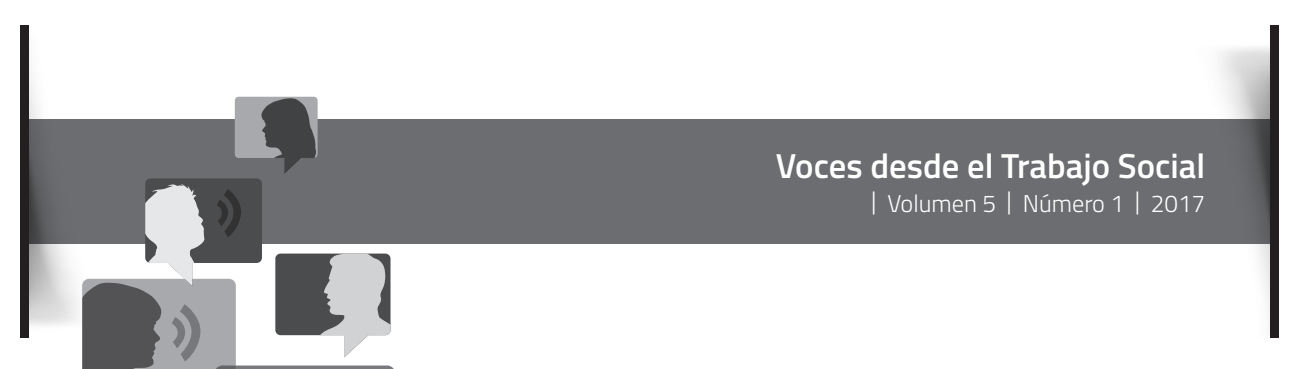

\title{
A PIE EN EL 100 X 35: SISTEMA DE TRANSPORTACIÓN COMO DETERMINANTE SOCIAL DE LA SALUD EN LA POBLACIÓN INFANTIL CON ENFERMEDADES CRÓNICAS
}




\section{Zaiska Pacheco Cruz}

Egresada de la Escuela Graduada de Trabajo Social Beatriz Lassalle de la Universidad de Puerto Rico. Labora como trabajadora social en el escenario de salud pública ofreciendo servicios a niñez con enfermedades crónicas del Hospital Pediátrico Universitario en San Juan.

\section{Recibido:}

10 de abril de 2017

Aprobado:

25 de agosto de 2017 


\section{A PIE EN EL $100 \times 35$ : SISTEMA DE TRANSPORTACIÓN COMO DETERMINANTE SOCIAL DE LA SALUD EN LA POBLACIÓN INFANTIL CON ENFERMEDADES CRÓNICAS}

escrito por of

Zaiska Pacheco Cruz

Resumen

Los retos que encara la población infantil con enfermedades crónicas han sido poco estudiados en Puerto Rico. Los servicios médicos especializados se concentran en la zona metropolitana. Ello plantea dificultades de acceso, en el contexto de un sistema de transportación que no satisface las necesidades de la población. Este artículo examina el impacto de las barreras de transportación en la continuidad del cuidado médico y la calidad de vida de la niñez con diagnósticos crónicos de salud. Describe las particularidades del sistema de transportación actual y las reacciones a los problemas sistémicos de movilidad y accesibilidad. Propone adoptar la perspectiva de los determinantes sociales de la salud para ampliar la discusión sobre las barreras de transportación y su vinculación con la exclusión social. Además, invita a replantearnos la transportación como derecho social. 
Descriptores: enfermedades crónicas, determinantes sociales de la salud, transportación, niñez, trabajo social.

\section{Abstract}

The hurdles faced by children with chronic illness in Puerto Rico have not been studied extensively. Specialized medical services are concentrated in the metropolitan area. This represents a challenge to health care access within the context of a transportation system which fails to address the needs of the population. The article examines the significance of these obstacles with regards to medical care continuity and the quality of life of children diagnosed with chronic illness. The attributes of the current transportation system, as well as the responses to its systemic mobility and access problems, are described. The work encourages the use of social determinants of health as a framework to broaden the discussion on transportation barriers and their links to social exclusion. It also proposes a reassessment of transportation as a civil right.

Keywords: chronic illnesses, social determinants of health, transportation, children, social work. 
La transportación se perfila nuevamente como un asunto importante en temática de derechos civiles. El enfoque de hoy no es obtener un asiento en la fila delantera del autobús, sino garantizar que el autobús nos lleve a donde necesitamos ir.

- Angela Glover Blackwell, Fundadora de PolicyLink ${ }^{4}$

Las palabras de Angela Glover (The Leadership Conference Education Fund, 2011) que dan inicio a este artículo, nos remontan al momento histórico en el que Rosa Parks se negó a ceder su asiento a un pasajero blanco en un autobús del servicio colectivo. Su accionar inspiró la derogación de la segregación racial en los espacios públicos y nutrió al movimiento de derechos civiles. Glover deja entrever que la transportación debe ser considerada un derecho. La ausencia de medios de transportación accesibles y efectivos condiciona la interacción social, las oportunidades y el acceso a servicios indispensables para la subsistencia. El actual sistema de transportación puertorriqueño constituye una barrera para el acceso a servicios de salud dirigidos a la atención de la población infantil con enfermedades crónicas. Este artículo examina las particularidades del sistema de transporte colectivo en la Isla y reflexiona sobre su configuración como determinante social de la salud para la niñez con diagnósticos crónicos.

\footnotetext{
$4 \quad$ Originalmente: "Transportation is back as a major civil rights issue. Today's focus is not on getting a seat at the front of the bus but on making sure the bus takes us where we need to go", según citado en The Leadership Conference Education Fund (2011, p. 1). Traducción de la autora de este artículo.
} 


\section{Enfermedades crónicas y niñez en Puerto Rico}

Las enfermedades crónicas se definen como diagnósticos de salud que se manifiestan a lo largo de la vida con mayor severidad y se caracterizan por ser raramente curables (División de Prevención y Control de Enfermedades Crónicas, 2014). En Puerto Rico, una de cada dos personas adultas ha sido diagnosticada con al menos una enfermedad crónica (Agencia EFE, 2017). Las condiciones cerebrovasculares, el cáncer, la diabetes, los trastornos respiratorios, las condiciones neuropsiquiátricas, las enfermedades musculares y digestivas, los trastornos genitourinarios, los diagnósticos congénitos y las enfermedades de la piel constituyen enfermedades crónicas (División de Prevención y Control de Enfermedades Crónicas, 2014). Las enfermedades crónicas pueden generar discapacidad si no son tratadas a tiempo o dependiendo de su severidad (Andersen, 2010). El género, la edad y la pobreza son elementos vinculados a mayor cantidad de muertes y discapacidad en personas con diagnósticos de enfermedades crónicas al compararse con otros grupos (División de Prevención y Control de Enfermedades Crónicas, 2014). Un $56.6 \%$ de las muertes reportadas en Puerto Rico para el 2012 fueron atribuidas a enfermedades crónicas (Rodríguez, 2014). El cáncer constituye la enfermedad crónica más mortal para la población puertorriqueña y la diabetes es una de las condiciones más comunes en la Isla (Departamento de Salud, 2017).

La incidencia de las enfermedades crónicas en la niñez puertorriqueña ha sido poco documentada y la limitada información disponible no se encuentra agrupada en una base de datos unitaria. La población infantil tampoco está representada en el perfil elaborado en el Plan de Acción de 
Enfermedades Crónicas para Puerto Rico 2014-2020 por el Departamento de Salud (División de Prevención y Control de Enfermedades Crónicas, 2014). Dicho informe se circunscribe a los grupos de 18 años o más con enfermedades crónicas. Existen sistemas de vigilancia para algunas enfermedades crónicas que pueden ofrecer datos sobre estas condiciones en la niñez de la Isla. Por ejemplo, el Registro Central de Cáncer de Puerto Rico, durante el período de 2008 a 2012, identificó un promedio de 152 diagnósticos de cáncer infantil y esta condición se constituyó como la quinta causa de muerte en ese grupo (Zavala-Zegarra, et al., 2015). La Fundación Pediátrica de Diabetes (2017) reporta que posee una membresía de alrededor de 2,500 que hace uso de sus servicios. Esto no representa el universo de la niñez con diagnóstico de diabetes.

En Estados Unidos, la tasa de niñez afectada por múltiples diagnósticos de enfermedad crónica es de uno en cada 15 (Andersen, 2010). En países latinoamericanos, los diagnósticos de enfermedades crónicas en la niñez han aumentado y se destacan entre los más comunes: el asma, los desórdenes del neurodesarrollo, las enfermedades congénitas, el cáncer, las condiciones cardiovasculares y la diabetes (Laborde et al., 2015).

Las condiciones crónicas implican un alto costo para los sistemas de salud y para las personas que las experimentan. Se estima que Puerto Rico invierte un 85\% del presupuesto de cuidado directo de salud en la atención de enfermedades crónicas (Vargas Vidot, 2017). Esta proporción es mayor cuando se compara con otras regiones. Estados Unidos invierte un $75 \%$ de su presupuesto de salud en la atención de las enfermedades crónicas (Ruiz, 2015) y en la Unión Europea se calcula un 80\% en este mismo renglón (European 
Chronic Disease Alliance, 2016). Un estudio comisionado por la Fundación Robert Wood Johnson (Andersen, 2010), reveló que la inversión de los sistemas de salud en una persona con enfermedad crónica es tres veces mayor al de una persona sin padecimientos crónicos. La atención de estas enfermedades está enmarcada por múltiples visitas a especialistas médicos, consumo de medicamentos y estudios. La Organización Mundial de la Salud (2017) sostiene que los elevados costos asociados al tratamiento de las enfermedades crónicas desplazan hacia los límites de pobreza a las personas que enfrentan los diagnósticos.

\section{Sistema de transportación en Puerto Rico Antecedentes del transporte colectivo}

A finales del siglo XIX, dio inicio la construcción de un ferrocarril con el propósito de facilitar el intercambio económico basado en el comercio de la caña de azúcar (Surillo, 2014). Para el 1924, como ilustra la Figura 1 (United States Department Of Commerce, 1924), el ferrocarril contaba con nueve rutas a través de la costa que se vinculaban a las centrales azucareras. La decadencia del comercio de azúcar y la competencia que representaban los vehículos de motor como medios de transporte abonaron al cierre de operaciones del ferrocarril en la década del cincuenta (Santamaría, 1994).

En la coyuntura del cierre del ferrocarril, el país experimentaba un proceso de industrialización con el proyecto Operación Manos a la Obra, junto al crecimiento poblacional y la migración interior. Este panorama generó la necesidad de construir más viviendas y convirtió al automóvil en el medio principal de transporte ante el desparramamiento urbano 


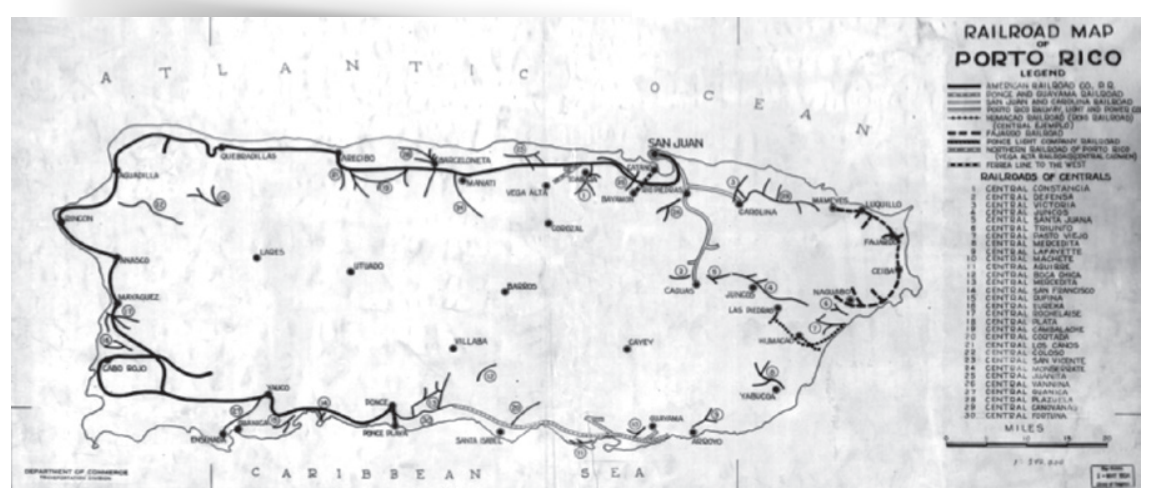

Figura 1. Mapa del ferrocarril en Puerto Rico. Reimpreso de United States Department Of Commerce. (1924). Railroad map of Porto Rico. Recuperado de https://www.loc.gov/item/98687137/ Imagen de dominio público.

(Aponte Meléndez, 2016). Durante la época de los cincuentas, despuntó la construcción de carreteras y expresos, por lo que el ferrocarril se sustituyó por el vehículo privado y los camiones (Crespo, 2010). La acelerada modernización del país y la carencia de planes para articular los espacios urbanos vino acompañada de congestión vehicular y ausencia de un sistema de transportación colectivo eficaz (Aponte Meléndez, 2016).

\section{Transporte colectivo actual}

El sistema de transportación colectivo en Puerto Rico está compuesto por la Autoridad Metropolitana de Autobuses (AMA), el Tren Urbano, guaguas públicas y el transporte marítimo. La concentración de estos servicios se ubica en siete pueblos de la zona urbana de la Isla y las dos islas municipio. Las únicas alternativas de transporte colectivo para las áreas fuera de la zona metropolitana son las deterioradas líneas de carros públicos (Luyanda Villafañe, 2011) y las guaguas públicas de la región centro-oriental de Caguas. 
La AMA fue creada mediante política pública en el 1959 con el propósito de proveer transportación en la región metropolitana (Gobierno de Puerto Rico, 2017). Actualmente, cuenta con 30 rutas de autobuses (que incluyen rutas expreso de MetroBus) que operan principalmente de lunes a sábado y cinco rutas de porteadores públicos que en conjunto cubren zonas de los municipios de San Juan, Carolina, Bayamón, Cataño, Loíza, Trujillo Alto y Toa Baja (Autoridad de Transporte Integrado, 2017). La población con diversidad funcional y condiciones de salud que no le permitan abordar un autobús o esperar en una parada, tiene disponible el programa Llame y Viaje, con rutas similares a las de la AMA. La AMA ha experimentado reducciones en rutas y horario de servicio ante ajustes fiscales.

El Tren Urbano opera a través de tres municipios del área metropolitana (San Juan, Bayamón y Guaynabo) con un total de 16 estaciones. El proyecto del Tren Urbano enfrenta hasta hoy día, críticas ante su elevado costo de construcción, limitada cobertura y fracaso en los estimados de utilización por pasajeros y pasajeras. Comenzó operaciones en el 2005 y su costo de construcción fue de \$2,250 millones (Departamento de Transportación y Obras Públicas, 2011). La cantidad de personas transportadas por el Tren Urbano se estima entre 38,000 a 40,000 y los fines de semana entre 12,000 y 15,000, por debajo de las proyecciones al inicio de su construcción que estimaban 110,000 anuales (Cintrón Arbasetti, 2014). El Tren Urbano carece de integración con rutas alimentadoras que hagan de este una alternativa más atractiva y efectiva al vehículo privado (Saker Jiménez, 2017).

La planificación del transporte colectivo en la Isla ha carecido de un enfoque centrado en la accesibilidad para la ciudadanía. Entendiendo la accesibilidad como "la capacidad 
de las personas para alcanzar los servicios y actividades que desean obtener o necesitan para subsistir" (Navarro, 2016), el sistema de transportación se ha quedado corto en su misión de comunicar al país y le ha fallado a la población al no adoptar la óptica del transporte como reductor de la desigualdad en acceso. Primero, el ferrocarril fue ideado para facilitar el intercambio comercial entre las centrales azucareras. Luego, la planificación de las carreteras se concibió para lidiar con el creciente número de vehículos de motor, y por lo tanto, en función del carro y en beneficio de las compañías automotrices.

\section{Uso de vehículos privados}

La sociedad puertorriqueña es altamente dependiente del transporte en vehículos de motor privados. Sólo un 7\% de los viajes realizados por la población puertorriqueña son a través de medios de transporte alternos al vehículo privado (Luyanda Villafañe, 2011). En el 2010, se registraron un total de 3,020,455 vehículos en la Isla, de los cuales, 2,311,686 eran automóviles privados (Departamento de Transportación y Obras Públicas, 2010). En este mismo año, Puerto Rico ocupó el lugar número 12 entre los países con mayor cantidad de automóviles por habitantes, superado por Guam, Italia, Malta, Nueva Zelanda, Australia, Luxemburgo, Islandia, Liechtenstein, Estados Unidos, Mónaco y San Marino (Primerahora.com, 2014). Aunque la sociedad puertorriqueña es altamente dependiente del vehículo privado, esto no se traduce en mayor movilidad o acceso. La proliferación de carros no significa: primero, que todas las personas tienen igual oportunidad económica para obtener y mantener un vehículo privado; y segundo, que los mismos estén en condiciones de uso. 
El hecho de que el principal medio de transportación en Puerto Rico es el automóvil, es una manifestación de la pobre utilización de los terrenos y la inefectividad de los medios de transporte colectivo. La fragmentación del servicio colectivo hace indispensable la posesión de un vehículo privado. Esto añade una gran carga económica. Ejemplo de ello, es que las familias de la región centro-oriental (Caguas y municipios adyacentes) de bajos ingresos gastan el 43\% de su presupuesto en transporte (Izquierdo Encarnación, 2016).

\section{Servicios para enfermedades crónicas infantiles y transportación desde la óptica de los determinantes sociales de la salud}

Los determinantes sociales de la salud son definidos como "las circunstancias en que las personas nacen, crecen, viven, trabajan y envejecen, y los sistemas establecidos para combatir las enfermedades" (Comisión sobre Determinantes Sociales de la Salud, 2008, p. 1). El análisis centrado en los determinantes sociales de la salud permite entender cómo incide el contexto en el cual se desarrolla la persona y su enlace con las oportunidades de salud. Se aleja de los argumentos simplistas que depositan la responsabilidad exclusiva en la figura de la persona, ignorando elementos estructurales que condicionan el nivel de salud. Las barreras en el acceso a servicios sanitarios y su relación con los determinantes sociales de la salud son mejor comprendidas si se inserta el análisis de los problemas de transportación (Williams, Ortiz \& Browne, 2014). Numerosos estudios sostienen que la ausencia de servicios de transportación efectivos afecta el acceso a servicios de salud para el tratamiento de las enfermedades crónicas (Cornelius 
et al., 2017; Roessler et al., 2013; Ruggiano, Shtompel, Whiteman \& Sias, 2017; Smith et al., 2017; Syed, Gerber \& Sharp, 2013). El transporte puertorriqueño se configura como determinante social de la salud en la medida en que limita las oportunidades de vinculación efectiva con los medios de subsistencia. El fragmentado transporte colectivo condiciona aún más el acceso a salud para la población que reside fuera del área metropolitana. Para ello es puntual, ofrecer una mirada a la distribución de los servicios de salud pediátricos en Puerto Rico.

Según Alameda (2013), la región Metro/Fajardo cuenta con el $52 \%$ del total de todas las especialidades médicas. La mayoría de los servicios especializados en salud infantil se ubican en el área metropolitana. La Isla cuenta con tres hospitales pediátricos localizados en los municipios de San Juan y Bayamón (Departamento de Salud, 2014). Las familias que residen fuera del área metropolitana experimentan una gran carga económica asociada a transportación. Este es el ejemplo de la niñez dependiente de diálisis en Puerto Rico. El único centro de diálisis infantil ubica en el Hospital Pediátrico Universitario del Centro Médico de Río Piedras. La población que recibe tratamiento en esta unidad visita las instalaciones con una frecuencia de tres a cuatro veces a la semana. Un ejercicio numérico puede ofrecer un panorama del gasto en transportación para esta población. Una familia residente de Añasco recorre 92 millas hacia San Juan, según la calculadora de millaje del Departamento de Transportación y Obras Públicas (2017). Bajo la presunción de que el vehículo consuma 20 millas por galón y tomando el precio actual de gasolina de 68 centavos, cada viaje tendría un costo de $\$ 11.84$ (ida y vuelta \$23.68). Si asistiera tres veces en semana a diálisis, la familia tendría que separar \$284.16 mensualmente para 
gasolina. La carga añadida a las finanzas familiares reduce las oportunidades de distribuir sus ingresos en otras necesidades vitales. Esta situación se reproduce en otros grupos infantiles con enfermedades crónicas.

A este panorama se añade la escasez de personal médico pediátrico, lo que precariza la disponibilidad de especialistas que puedan atender e identificar las señales tempranas de complicaciones de salud y de las enfermedades crónicas infantiles. La población de pediatras en la práctica activa, una especialidad que ha experimentado un constante descenso, se estima entre 750 a 800 (Sociedad Puertorriqueña de Pediatría, 2016). En el 2011, se calculó que ocho municipios no tenían pediatras con oficina privada, generando visitas a hospitales de niñez en búsqueda de este servicio (Sociedad Puertorriqueña de Pediatría, 2016). En otras especialidades de pediatría, para el período de 2007 a 2010, se registraron practicando: tres especialistas de nefrología, cinco ortopedas, siete en cirugía y una persona en endocrinología, al igual que en oftalmología (Departamento de Salud, 2013). Según Víctor Ramos, Presidente del Colegio de Médicos-Cirujanos de Puerto Rico, la Isla presenta una escasez de especialistas cuya magnitud provoca que la espera para obtener citas médicas sea de hasta un año (Coto, 2016). Para la niñez diagnosticada con una condición crónica de salud, la espera para una evaluación médica puede traducirse en complicaciones severas a la salud y uso frecuente de las salas de emergencia. Si la familia no cuenta con transportación efectiva y confiable para asegurar las visitas médicas, se prevé un riesgo aumentado para su vida.

La escasez y pobre distribución geográfica de los servicios especializados en salud pediátrica coloca a la población infantil en una situación de mayor desventaja. A esto se añade, que las personas con limitados ingresos económicos son las que 
más enfrentan barreras de transportación (Syed, Gerber \& Sharp, 2013). El 84\% de la niñez residente en Puerto Rico vive en zonas de altos niveles de pobreza (The Annie E. Casey Foundation, 2017). Un 57.1\% de la población infantil se encuentra bajo el nivel de pobreza y la mayoría de los pueblos más pobres se ubican en la zona central y oeste del país (Instituto del Desarrollo de la Juventud, 2017). Precisamente, estas zonas encaran los mayores desafíos al momento de identificar fuentes de transportación consistentes y efectivas. Lucas (2012) plantea la vinculación del concepto de exclusión social aplicado a los problemas de transportación. Bajo dicha conceptualización, la niñez con enfermedades crónicas enfrenta exclusión por transporte.

Las dificultades asociadas a transportación para acceder servicios de salud entrañan otras problemáticas que impactan la continuidad del cuidado médico y el manejo exitoso de las enfermedades crónicas. Una investigación realizada en Estados Unidos con personas adultas dependientes de diálisis (Moist et al., 2008) evidenció una correlación entre los viajes prolongados para llegar a los tratamientos con un aumento en el riesgo de mortalidad y una disminución de la calidad de vida. Otro estudio con población en diálisis, identificó que los problemas de transportación también inciden en la adherencia al tratamiento y en aumentar los estresores por dificultades para asistir a las terapias (Park \& Kear, 2017). La inaccesibilidad de transportación combinada con la distancia geográfica de los servicios de salud se traduce en abandono del tratamiento, diagnósticos tardíos y posposición de citas médicas. En Inglaterra, se estimó que cerca de 1.5 millones de personas se ausentaron a citas o no buscaron atención médica tras enfrentar barreras de transportación (Social Exclusion Unit, 2003). 
Por otro lado, Williams, Ortiz y Browne (2014) hallaron en su estudio con pacientes de lupus, que las complicaciones vinculadas a pobre acceso a transportación limitan la participación de las personas en el tratamiento. En la situación de la niñez con enfermedades crónicas, las barreras en transportación y la distancia que recorren para obtener los servicios, además, afectan su integración a otras áreas importantes de la vida. El desempeño de las tareas propias de la niñez se ve trastocado ante asistencia irregular a clases, limitados intercambios con pares y disminución del tiempo de ocio con la familia (Pacheco Cruz, 2015).

\section{Reacciones ante el problema de transportación y acceso a servicios de salud pediátrica}

El problema sistémico de la transportación en Puerto Rico ha recibido respuestas insuficientes. Algunos municipios han implementado programas de transportación a citas médicas, especialmente para facilitar el transporte desde pueblos distantes hasta el Centro Médico de Río Piedras. La disponibilidad de este tipo de programa depende de la salud fiscal de los pueblos y está limitado al horario de operación de los municipios. No siempre puede parear efectivamente las necesidades de las personas que solicitan el servicio y los días en que se otorgan las citas. Es de conocimiento personal, clínicas asociadas al Centro Médico de Río Piedras que atienden un alto volumen de pacientes, cuyas operaciones se extienden luego de las 5:00 p.m., horario en el que típicamente ya no está en función el transporte provisto por los municipios

Por otro lado, se ha generalizado el uso del transporte no emergente. Este transporte consiste en entidades privadas que ofrecen transportación a citas médicas mediante el pago 
de una tarifa o a través de la aprobación de planes médicos. La transportación no emergente es un servicio que no forma parte de las cubiertas básicas de los planes médicos y es aprobado mediante excepción. Tener un diagnóstico de enfermedad crónica no garantiza la aprobación del transporte no emergente, lo que añade sentimientos de incertidumbre e impotencia.

Reconociendo que los problemas de transportación son una barrera para la continuidad del tratamiento médico, algunos programas de trasplante pediátrico en la Isla solicitan a pacientes que presenten evidencia de un fondo económico para costear gastos post-trasplante, entre ellos la transportación. Esto se traduce en que las familias llevan a cabo actividades y colectas para recaudar dinero. En dichos procesos, se ven obligadas a compartir información médica que en otros contextos no divulgarían. La solicitud de un fondo económico para sufragar gastos de transportación surge como respuesta ante un país que carece de unos modos efectivos de transportación. Construir ese fondo económico para familias que ya son golpeadas por los gastos vinculados a enfermedades crónicas, representa mayores estresores, las coloca en posiciones vulnerables y puntualiza las disparidades en la salud.

\section{A modo de reflexión}

La atención efectiva de las enfermedades crónicas infantiles requiere del accionar sobre los determinantes sociales de la salud. La transportación en Puerto Rico constituye una barrera en el alcance de mejores oportunidades de salud y acceso a servicios esenciales para incrementar la calidad de vida de la población infantil con enfermedades crónicas. La capacidad económica para pago, la localización geográfica y la 
distribución de las rutas del servicio colectivo son elementos que condicionan la movilidad en el país. Se hace urgente conducir investigaciones que posibiliten una mejor comprensión sobre el alcance de los diagnósticos crónicos de salud en la niñez y el impacto de las barreras de transportación.

Puerto Rico no ha logrado articular una política de transportación que concretice la movilidad y accesibilidad como un asunto de justicia. Es imperativo desarrollar un proyecto de transportación que vislumbre la comunicación y conexión a lo largo y ancho del país. El argumento de la pobre salud fiscal del país, ha sido uno de los escollos para abandonar los planes de un sistema de transporte inclusivo. Ante ello, debemos recordar que no contar con una infraestructura de transportación accesible y efectiva genera un alto costo humano. La unión de voluntades para reconfigurar los medios de transportación del país, es necesaria para atacar la raíz del problema y abandonar medidas remediales que le colocan parchos al sistema encubriendo las inequidades en la salud. La inversión en infraestructura de transportación es vital para construir sociedades más inclusivas y sostenibles acompañadas por el empoderamiento de las personas (Organización de las Naciones Unidas, 2015). Las experiencias exitosas de las reformas de transporte implementadas en países como Australia (Lucas, 2012) e Inglaterra (Social Exclusion Unit, 2003), dan cuenta de la importancia de adoptar la planificación participativa, la perspectiva de los determinantes sociales de la salud y el concepto de la exclusión social en el diseño de políticas de transportación para atender las desigualdades. Estar a pie en el 100 x 35 implica exclusión y riesgo para la niñez con enfermedades crónicas. Este panorama exige repensar la transportación no como un servicio, sino como un derecho social. 


\section{Referencias}

Agencia EFE. (15 de marzo de 2017). Uno de cada dos adultos vive con al menos una dolencia crónica. El Nuevo Día. Recuperado de http://www.elnuevodia.com/

Alameda, José. (2013). The Demand and Supply of Physicians in Puerto Rico: Historical trends and forecasting 2013 to 2019. San Juan: Departamento de Salud. Recuperado de http://www.salud.gov.pr/Estadisticas-Registros-yPublicaciones/Pages/Publicaciones-sobre-la-salud.aspx. Recuperado de http://www.salud.gov.pr/EstadisticasRegistros-y-Publicaciones/Pages/Publicaciones-sobre-lasalud.aspx

Andersen, Gerard. (2010). Chronic Care: Making the Case for Ongoing Care. Princeton, N.J.: Robert Wood Johnson Foundation.

Aponte Meléndez, Sara. (2016). La vivienda y movilidad de San Juan y ciudad de Panamá entre 1940 y 1960: análisis histórico de sus políticas públicas de cara al siglo XX1. PLERUS, 26, 209-229.

Autoridad de Transporte Integrado. (2017). Rutas y mapas. Recuperado de https://ati.pr/rutas-y-mapas/

Cintrón Arbasetti, Joel. (18 de julio de 2014). Un viaje en tren de Sagrado Corazón a Bayamón a las nueve de la noche. 80grados. Recuperado de http://www.80grados.net

Comisión sobre Determinantes Sociales de la Salud. (2008). Conceptos clave. Recuperado de http://www.who.int/ social_determinants/thecommission/finalreport/key_ concepts/es/

Cornelius, Talea; Jones, Maranda; Merly, Cynthia; Welles, Brandi; Kalichman, Moira \& Kalichman, Seth. (2017). Impact 
of food, housing, and transportation insecutiry on ART adherence: A hierarchical resources approach. AIDS Care, 9(4), 449-457. doi:10.1080/09540121.2016.1258451

Coto, Danica. (27 de octubre de 2016). Crisis económica provoca éxodo masivo de doctores en Puerto Rico. The Associated Press. Recuperado de http://cb.pr/author/ associated-press/

Crespo, Gisela. (5 de noviembre de 2010). De San Juan a Ponce en tren, como antes. 80grados. Recuperado de http://www.80grados.net/

Departamento de Salud. (2013). Informe estadístico de los profesionales de la salud. Undécimo registro 20072010. San Juan: Secretaría Auxiliar de Planificación y Desarrollo. Recuperado de http://www.salud.gov.pr/ Estadisticas-Registros-y-Publicaciones/Publicaciones/ Profesionales\%20de\%20la\%20Salud\%202007-2010.pdf

Departamento de Salud. (2014). Informe de la salud en Puerto Rico. San Juan: Autor.

Departamento de Salud. (2017). División para la Prevención y Control de Enfermedades Crónicas (DPCEC). Recuperado de http://www.salud.gov.pr/Sobre-tu-Salud/Pages/ Condiciones/Enfermedades-Cronicas.aspx

Departamento de Transportación y Obras Públicas. (2010). Registro de vehículos de motor por municipios y por categorías correspondientes al año fiscal del 2009-2010. Recuperado de http://www.estadisticas.gobierno.pr/ iepr/Estadisticas/InventariodeEstadisticas/tabid/186/ctl/ view_detail/mid/775/report_id/54d5b157-65c7-499b-98fff6275d95fc69/Default.aspx?f=1.3,1.4,2 
Departamento de Transportación y Obras Públicas. (24 de mayo de 2011). El Rol de la Alternativa de Transporte Integrado y los Sistemas de Autobuses en el AMSJ. Ponencia presentada en el Primer Simposio Formando Ciudades Habitables: el futuro del transporte colectivo en Puerto Rico celebrado en el Colegio de Ingenieros y Agrimensores de Puerto Rico, San Juan: PR. Recuperado de https://academic.uprm.edu/ amfigueroa/Presentations/ PDF_Spring2011/ATI.pdf

Departamento de Transportación y Obras Públicas. (2017). Calculadora de millaje. Recuperado de http://www.dtop. gov.pr/carretera/det_content.asp?cn_id=24

División de Prevención y Control de Enfermedades Crónicas. (2014). Plan de acción de enfermedades crónicas para Puerto Rico 2014-2020. San Juan: Departamento de Salud.

European Chronic Disease Alliance. (2016). ECDA Call to Action for a Comprehensive EU Framework on Chronic Diseases by 2017. Recuperado de http://www.alliancechronicdiseases. org/home/

Fundación Pediátricade Diabetes. (2017). Serviciosyactividades. Recuperado de http://www.fundacionpediatricadiabetes. org/

Gobierno de Puerto Rico. (2017). Autoridad Metropolitana de Autobuses (Dpto.DTOP). Recuperado de http://www2. pr.gov/Directorios/Pages/InfoAgencia.aspx?PRIFA=174

Instituto del Desarrollo de la Juventud. (2017). Municipality Index: Children and Teenagers Under 18 Years Below the Poverty Level. Recuperado de http://juventudpr.org/en/ municipality-index/ 
Izquierdo Encarnación, José. (26 de octubre de 2016). Financiamiento de la estructura vial y de transportación. Ponencia presentada en la Segunda Conferencia Anual Dr. Hermenegildo Ortiz Quiñones Movilidad y Equidad: retos para la planificación y política pública sobre el transporte sustentable en Puerto Rico, San Juan: PR. Recuperado de https://www.youtube.com/ watch?v=FrX5KupK8yQ\&t=5111s

Laborde, Amalia; Tomasina, Fernando; Bianchi, Fabrizio; Bruné, Marie-Noel; Buka, Irena; Comba, Pietro,... Landrigan, Philip. (2015). Children's health in Latin America: The influence of environmental exposures. Environmental Health Perspectives, 123(3), 201-209. doi:10.1289/ehp.1408292

Lucas, Karen. (2012) Transport and social exclusion: Where are we now? Transport Policy, 20, 105-113. doi:10.1016/j. tranpol.2012.01.013

Luyanda Villafañe, Felipe. (24 de mayo de 2011). La necesidad del transporte colectivo y el desarrollo en Puerto Rico. Ponencia presentada en el Primer Simposio Formando Ciudades Habitables: el futuro del transporte colectivo en Puerto Rico celebrado en el Colegio de Ingenieros y Agrimensores de Puerto Rico. San Juan: PR. Recuperado https://academic.uprm.edu/ amfigueroa/Presentations/ PDF_Spring2011/Luyanda\%20tp\%20colectivo.pdf

Moist, Louise; Bragg-Gresham, Jennifer; Pisoni, Ronald; Saran, Rajiv; Akiba, Takashi; Jacobson, Stefan;...Port, Friedrich. (2008). Travel Time to Dialysis as a Predictor of HealthRelated Quality of Life, Adhrence, and Mortality: The Dialysis Outcomes and Practice Patterns Study (DOPPS). American Journal of Kidney Diseases, 51(4), 641-650. doi: 10.1053/j. ajkd.2007.12.021 
Navarro, Criseida. (26 de octubre de 2016). Geografía de la desigualdad (pobreza, salud, educación). Ponencia presentada en la Segunda Conferencia Anual Dr. Hermenegildo Ortiz Quiñones: Movilidad y equidad: retos para la planificación y política pública sobre el transporte sustentableenPuertoRico,SanJuan:PR. Recuperado https:// www.youtube.com/watch?v=FrX5KupK8yQ\&t=5111s

Organización de las Naciones Unidas. (2015). Transformar nuestro mundo: la Agenda 2030 para el Desarrollo Sostenible. Recuperado de http://unctad.org/meetings/es/ SessionalDocuments/ares70d1_es.pdf

Organización Mundial de la Salud. (2017). 10 datos sobre las enfermedades no transmisibles: cifras y datos. Recuperado dehttp://www.who.int/features/factfiles/noncommunicable_ diseases/es/

Pacheco Cruz, Zaiska. (30 de octubre de 2015). Población infantil con enfermedades crónicas en Puerto Rico: implicaciones del Modelo de Cuidados Crónicos Expandido para la práctica del trabajo social. Ponencia presentada en el Segundo Congreso Internacional del Colegio de Profesionales del Trabajo Social de Puerto Rico, El Derecho a la salud y las políticas sociales: desafíos para el Trabajo Social, San Juan: PR.

Park, Seri \& Kear, Tamara M. (2017). Current state-of-practice: Transportation for patients with end stage renal disease. Nephrology Nursing Journal, 44(4), 309-315.

Primerahora.com. (3 de junio de 2014). Puerto Rico en los primeros países de más carros por habitantes. Primera Hora. Recuperado de http://www.primerahora.com 
Rodríguez, Idania. (2014). Informe de enfermedades crónicas, Puerto Rico 2012. San Juan, PR.: Departamento de Salud.

Roessler, Richard T.; Bishop, Malachy; Rumrill, Phillip D.; Sheppard-Jones, Kathleen; Waletich, Brittany; Umeasiegbu, Veronica \& Bishop, Lisa. (2013). Specialized housing and transportation needs of adults with multiple sclerosis. Work, 45(2), 223-235. doi:10.3233/WOR-2012-1455

Ruggiano, Nicole; Shtompel, Natalia; Whiteman, Karen \& Sias, Kathy. (2017). Influences of Transportation on Health Decision-Making and Self-Management Behaviors among Older Adults with Chronic Conditions. Behavioral Medicine, 43(1), 61-70. doi:10.1080/08964289.2015.1065788

Ruiz, Gloria. (21 de marzo de 2015). Mueren los boricuas por enfermedades prevenibles. El Nuevo Día. Recuperado de http://www.elnuevodia.com

Saker Jiménez, Gabriela. (26 de marzo de 2017). Al Tren Urbano le faltan las extremidades. El Nuevo Día. Recuperado de http://www.endi.com

Santamaría, Antonio. (1994). Los ferrocarriles de servicio público en Puerto Rico (1870-1990). Revista Complutense de Historia de América, 20, 207-228.

Smith, Mathew L.; Prohaska, Thomas R.; MacLeod, Kara E.; Ory, Marcia G.; Eisenstein, Amy R.; Ragland, David R.;... Satariano, William A. (2017). Non-Emergency Medical Transportation Needs of Middle-Aged and Older Adults: A Rural-Urban Comparison in Delaware, USA. International Journal of Environmental Research and Public Health, 14(2), 1-13. doi:10.3390/ijerph14020174 
Social Exclusion Unit. (2003). Making the Connections: Final Report on Transport and Social Exclusion. London: UK: Office of the Deputy Prime Minister. Recuperado de http://www. ilo.org/wcmsp5/groups/public/@ed_emp/@emp_policy/@ invest/documents/publication/wcms_asist_8210.pdf

Sociedad Puertorriqueña de Pediatría. (2016). Entrevista al Dr. Gerardo Tosca: las visitas preventivas en la oficina del pediatra son importantes para el desarrollo de los niños [Suplemento Pediatría]. Galenus, 57(2), 64-66. Recuperado de http://www.galenusrevista.com/

Surillo, Gricel. (2014). La llegada del ferrocarril a Puerto Rico. En Enciclopedia de Puerto Rico. Recuperado de http:// www.enciclopediapr.org

Syed, Samina; Gerber, Ben \& Sharp, Lisa. (2013). Traveling Towards Disease: Transportation Barriers to Health Care Access. Journal of Community Health, 38(5), 976-993. doi:10.1007/s10900-013-9681-1

The Annie E. Casey Foundation. (2017). 2017 KIDS COUNT Data Book. State Trends in Child Well Being. Baltimore: Autor. Recuperado de http://stg.juventudpr.org/wp-content/ uploads/2017/06/Kids-Count-Data-Book-20171.pdf

The Leadership Conference Education Fund. (2011). The Road to Health Care Parity: Transportation Policy and Access to Health Care. Recuperado de http://civilrightsdocs.info/pdf/ docs/transportation/The-Road-to-Health-Care-Parity.pdf

United States Department Of Commerce. (1924). Railroad map of Porto Rico. Recuperado de https://www.loc.gov/ item/98687137/ 
Vargas Vidot, José. (24 de marzo 2017). Voy abriendo camino... el foro. Evento para los profesionales de la salud renal en Puerto Rico. Conversatorio celebrado en el Hospital Auxilio Mutuo en San Juan, P.R.

Williams, Edith; Ortiz, Kasim \& Browne, Teri. (2014). Social determinants of health, the Chronic Care Model, and Systemic Lupus Erythematosus. International Journal of Chronic Diseases, 2014, 1-7. doi:10.1155/2014/361792

Zavala-Zegarra, Diego; Tortolero-Luna, Guillermo; TorresCintrón, Carlos; Alvarado-Ortiz, Mariela; Traverso-Ortiz, Maricarmen; Román-Ruiz, Yadira \& Ortiz-Ortiz, Karen J. (Eds.). (2015). Cáncer en Puerto Rico: 2008-2012. Incidencia y mortalidad. San Juan: Registro Central de Cáncer. 